\title{
Full spectrum neurorestoratology: enhancing neuroresponse to disasters
}

\author{
This article was published in the following Dove Press journal: \\ Journal of Neurorestoratology \\ 21 July 2014 \\ Number of times this article has been viewed
}

\author{
Russell J Andrews' \\ Leonidas Quintana ${ }^{2}$ \\ 'Ames Research Center, \\ Nanotechnology and Smart Systems, \\ National Aeronautics and Space \\ Administration, Moffett Field, CA, \\ USA; ${ }^{2}$ Department of Neurosurgery, \\ Valparaiso University School of \\ Medicine, Valparaiso, Chile
}

\begin{abstract}
With more than 200,000 deaths in some years from earthquakes alone, disasters, both natural and manmade, are a major challenge for neurorestoratology. To minimize permanent neurological injury and death, it is essential for treatment to begin immediately, within minutes ideally, but certainly within 24 hours. Fortunately, the humanitarian and medical response to disasters removes the socioeconomic, legal, and political barriers that can hinder the treatment of neurological disorders under normal (nondisaster) situations. Here we review the resources and equipment already available as well as in development to enhance prompt treatment of neurological injuries arising from disasters. To be sustainable, the response to disasters must be integrated into the ongoing daily health care delivery systems worldwide, from medical education and specialty training (resident/registrar) to acute and subacute intensive care to long-term rehabilitation. The "trauma center" concept developed in the USA and elsewhere for nonmass casualty response is an example of a program developed within the existing health care training and delivery infrastructure. We therefore propose a model for worldwide disaster response that integrates disaster neurorestoratology into health care delivery systems worldwide, both governmental and nongovernmental, and national and international. An overall blueprint is presented for the full spectrum of disaster neurorestoratology, from prevention of nervous system injury, to comprehensive and immediate acute care, to long-term neurorehabilitation. Such a comprehensive response to disasters would overcome the geographic, socioeconomic, and political barriers that presently impair our ability to respond effectively to the nervous system devastation caused by disasters of all types.
\end{abstract}

Keywords: disaster response, global health care, mobile hospitals, neurotrauma, telemedicine

\section{Introduction}

Trauma centers have evolved in developed countries to minimize the morbidity and mortality of common injuries, most commonly road traffic accidents, but in some societies other causes such as falls and gunshot injuries are common. Although injury prevention, in various forms ranging from modification of human behavior (eg, education regarding the risks of speeding and driving while intoxicated, and penalties for failing to observe safe practices) to safer equipment (eg, seat belts in cars and helmets for motorcyclists, bicyclists, and sports at risk for head injury), is clearly the ideal situation, disasters by definition are unpredictable, widespread, and disruptive of normal medical resources.

Although trauma centers have proven very effective at reducing the morbidity and mortality from injuries suffered by a small number of individuals at any one time, they are resource-intensive, in that elaborate medical facilities and full staffing 
must be immediately available at all times, ie, "24/7". ${ }^{1}$ The basic infrastructure necessary for a trauma center to function must also be available at all times, most obviously electricity, water, and food for the staff. All of these "givens" for a trauma center are likely to be disrupted in a disaster, and all must be imported immediately to translate the benefit that the trauma center provides for individual or small group injuries into a similar benefit for disasters, which may affect dozens to thousands of individuals. Moreover, unlike the fixed site "bricks and mortar" trauma center (to which individual trauma victims are rapidly transported by ambulance or helicopter), the medical response to a disaster must be entirely portable (most likely by helicopter) on very short notice (minutes to a few hours).

Unfortunately, unlike the trauma center response to individuals suffering injuries, the medical response to most disasters is measured in days to weeks in most instances. For the nervous system, such a delayed response is almost always an exercise in futility, in that gravediggers and nursing homes are of more benefit than a neurosurgeon to the patient who has suffered a nervous system injury when the response is delayed for days to weeks. To improve the response to disasters, we need to develop a new paradigm. The rapidity of intervention provided by the emergency response land and air ambulances and fixed site trauma centers needs to occur with similar rapidity in disasters. This paper presents a proposal for improving disaster response, and suggests the multiple additional benefits that will accrue when such a system is implemented on an international basis.

Only an outline of such a broad disaster response program can be presented in a single journal paper. However, the examples provided here can be elaborated and integrated into a comprehensive disaster response program. Since disasters rarely respect national borders, such a program will of necessity be a regional (ideally global) project. This is actually a huge advantage, given that disasters typically evoke a humanitarian response that transcends political, economic, religious, and national boundaries.

One international organization that would be integrated with this disaster response project is the United Nations Office for the Coordination of Humanitarian Affairs (UNOCHA). ${ }^{2}$ It is the responsibility of UNOCHA "to ensure a coherent response to emergencies", ie, to coordinate civilian and military response efforts as well as to monitor "global emergency relief stockpiles on behalf of the whole humanitarian community". However, the project described here is quite distinct from the role of UNOCHA in disaster response. Rather than serving as a coordinating body for different international, national, and local organizations relevant to disaster relief (the role of UNOCHA), the disaster relief project would be an integral part of the ongoing health care delivery and health personnel education system. This is similar to the difference between programs such as trauma centers and stroke centers (which are integrated with and indistinguishable from the health care education and delivery systems) and agencies ranging from Cooperative for Assistance and Relief Everywhere, Doctors Without Borders, Interaction, the Red Cross, to UNOCHA (which are external organizations designed to provide targeted relief for disasters and other situations of acute need). ${ }^{3}$

An analogy may help to clarify the difference between disaster relief agencies, the primary role of which is to respond to a disaster (one might call it an externally derived "treatment" for the disaster), and the disaster relief program proposed here, which is integrated into the ongoing health care delivery of (ideally) every country worldwide. As a hypothetical example, a person who has developed (or who was born with) a thyroid hormone deficiency might be given an externally supplied supplement (levothyroxine) to make up for the deficiency. An alternative approach would be to use gene therapy to repair the person's own thyroid hormone generation and regulation system to make up for the deficiency. This crude analogy points out the difference between an externally based disaster response system, that is solely charged with responding to disasters, and a disaster response system that is an integral part of the ongoing health care delivery system. One can argue that, by integrating the disaster response into the health care system (delivery, education, research), the long-term benefits to society as a whole would be much greater (and more efficient) than a one-time external response, much as repairing a defective thyroid hormone gene would be more effective than giving thyroid supplements for the rest of a person's life.

The heads of the World Health Organization and the World Bank Group recently proposed that universal health care be introduced worldwide as a means of not only improving global health but also as a mechanism for reducing global poverty. ${ }^{4}$ The authors assert that the doctor-patient relationship ("the bedrock of the global health equity movement") is the foundation upon which global resources (such as the World Health Organization and the World Bank) are addressed to provide universal health care. Health care professionals must take the lead on behalf of the entire 
population to see that universal health care becomes a reality worldwide. This means the physician must put the patient's health ahead of short-term personal financial gain in those countries where health care delivery is tied to charges to the patient for physician and hospital services.

In disaster relief, a similar need exists for the disasterstricken country to seek resources worldwide immediately to address the response. Perhaps the best example of global cooperation in disaster response is the Copiapó mining accident that occurred in northern Chile on August 5, 2010, less than 6 months after the devastating earthquake and tsunami that struck near the city of Concepción in southcentral Chile. At Copiapó, 33 miners were trapped 2,300 feet below the surface in the San José copper-gold mine; 17 days after the accident, it was discovered that the 33 miners were in fact alive in an underground shelter. The approach taken by the Chilean government, which, as a result of the Concepción earthquake and tsunami, has dedicated part of its military resources to disaster response, included rapid mobilization of the following resources: virtually every Chilean government ministry, three international drilling rig teams and more than a dozen multi-national corporations, and the US National Aeronautics and Space Administration (NASA). After 69 days trapped underground, this global rescue effort brought all 33 miners safely to the surface on October $13,2010 .^{5}$

The time has come for the global medical community to address disaster response through global cooperation in medical care delivery, medical education, and social service organizations of all types (both government and nongovernment, civilian and military).

Research organizations such as the International Association of Neurorestoratology and the Global College of Neuroprotection and Neuroregeneration, unlike clinical medical societies for the most part, are not inhibited by national boundaries. The nervous system is essentially identical for individuals worldwide, and our efforts to understand the nervous system and restore its function after injury frequently foster collaborations that similarly are not inhibited by national boundaries.

This paper is structured as follows: after describing the issues raised by disasters that are not typically present in situations of individual or small group injury, some of the resources and equipment available (or in development) that can greatly improve disaster response are presented. Ways in which the personnel needed for disaster response can be addressed are then considered. Finally, the advantages of such a comprehensive disaster response program for not only reducing the neuromorbidity and mortality caused by disasters, but also for improving both neurorestoratology research in particular and medical education and worldwide health care in general are discussed.

The neurorestoratology community can be instrumental in transforming disaster response into a mechanism for enhancing not only nervous system function following disasters but also the structure of health care delivery worldwide.

\section{Challenge of disaster response}

Whether natural (eg, earthquakes, typhoons, and tsunamis) or manmade (terrorist bombings and large-scale accidents, such as airplane accidents or collapsed buildings), disasters may not only damage the nervous systems (and other systems, eg, vascular, pulmonary, musculoskeletal) of large numbers of individuals, but they may also damage or destroy the medical infrastructure in the region affected by the disaster. A neurosurgeon without basic neuroimaging, an operating room, and support staff is useless. Not only are medical personnel needed (bearing in mind that medical personnel in the disaster zone are not themselves immune from incapacitation or death from the disaster), but the medical facilities and infrastructure needed to run the operating room must also be imported.

Some of the equipment needs for disaster response include:

- operating rooms (including anesthesia, instruments, sterilization)

- electricity (generators)

- water, nutrition, sanitation, and accommodation for personnel and patients

Some of the personnel needs for disaster response include:

- operating rooms (anesthesiologist, surgeon, nurses, support staff)

- supply teams (transporters, likely helicopter in most situations, and logistics)

- social service and rehabilitation (for postoperative care of patients).

In summary, not only are medical resources such as hospitals and doctors, and local infrastructure such as clean water and electricity, likely to be unavailable in a major disaster, but the access routes for supplies (notably highways, railways, and airports) are likely to be destroyed or unserviceable in the period immediately following a disaster. For an adequate response to a major disaster that will improve neurological 
outcomes, one must be able to import all necessary resources, usually by helicopter.

\section{Resources for disaster response}

The military bodies of many larger developed countries have assembled resources for immediate response to medical emergencies during times of conflict. In the USA, the US Army has developed the mobile emergency unit, a cargo container which, in various configurations, can serve as a self-contained operating room, recovery room, and patient ward. Combined mobile emergency units make up a combat support hospital, which parallels the civilian need for immediate medical resources in a disaster. The mobile emergency units can be transported by helicopter (Figure 1).

The recent technological advances in portable medical equipment to support disaster response are impressive. Available presently are lightweight devices for the transport of liquid oxygen and its conversion to gaseous oxygen for patient use (Figure 2A), mass oxygen distribution systems for providing oxygen for up to 150 casualty patients simultaneously (Figure 2B), and liquid oxygen generators for use in harsh remote environments (Figure 2C). For patient monitoring and cardiac defibrillation, small and lightweight devices are also available (an example of which is shown in Figure 3) that in addition to defibrillation can simultaneously monitor a 12-lead electrocardiogram, noninvasive blood pressure, endtidal $\mathrm{CO}_{2}$, oxygen saturation, two temperature channels, and three invasive pressures, with a 6-hour battery life.

In mass casualty situations, computerized tomography (CT) scanning is crucial in the evaluation and treatment of patients who have suffered injuries to the nervous system. A $400 \mathrm{~kg}$ portable head CT scanner $\left(\right.$ CereTom $\left.^{\circledR}\right)$ has been

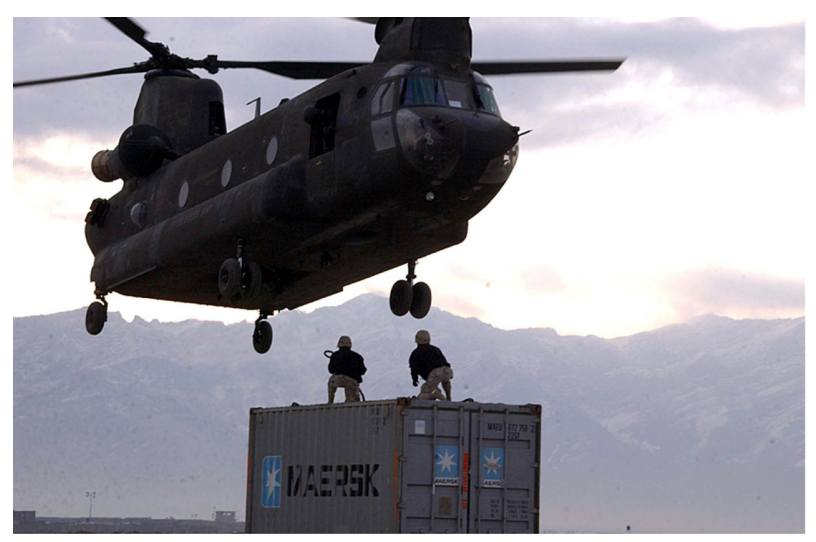

Figure I Chinook helicopter used by the US Army and other organizations for transport of cargo containers that can serve as portable operating rooms and other facilities necessary for a disaster response hospital. This helicopter has a payload of $12,000 \mathrm{~kg}$ and a range of $\mathrm{I}, 100 \mathrm{~km}$.
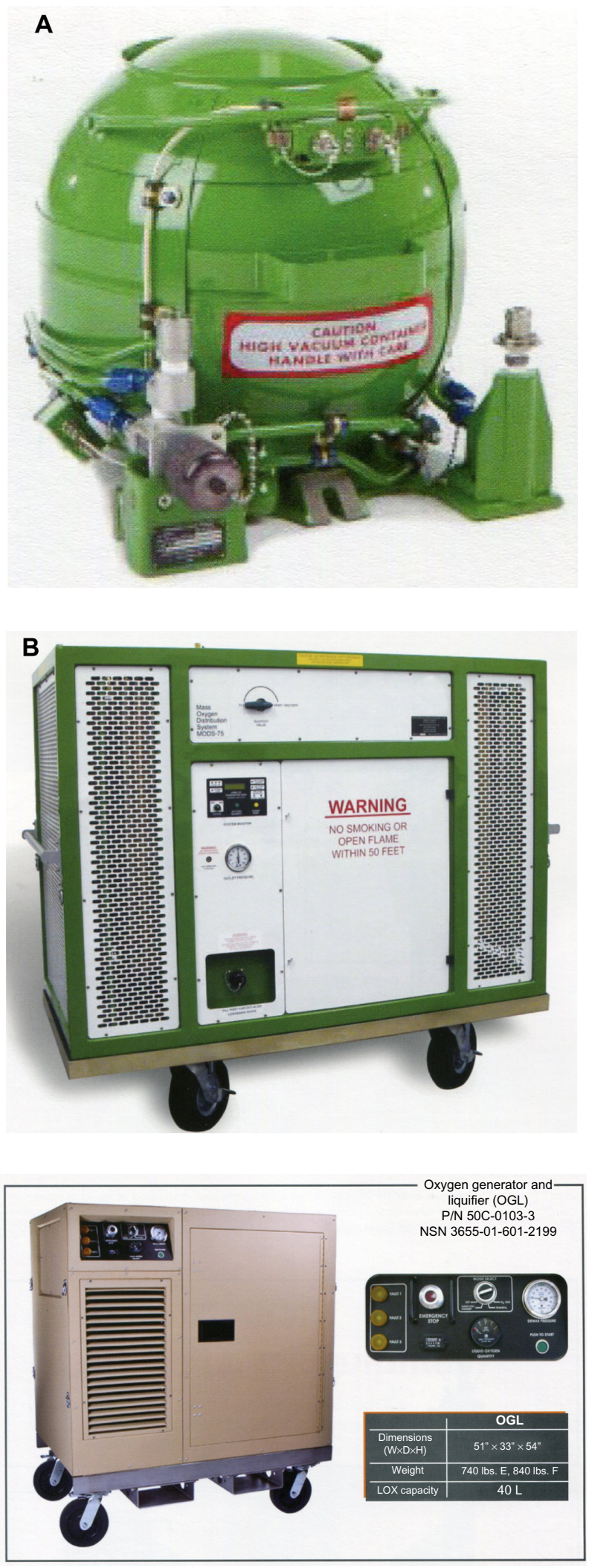

Figure 2 (A) Lightweight (20 kg full) portable liquid oxygen convertor for medical evacuation use in ambulances and helicopters. (B) Portable liquid oxygen storage and gaseous distribution system that can supply $64,500 \mathrm{~L}$ of gaseous oxygen at a flow rate of up to $450 \mathrm{~L}$ per minute (ie, $3 \mathrm{~L}$ per minute for 150 patients for nearly 2.5 hours). (C) Liquid oxygen generator that can be used to refill (A) or (B) and generates I L of liquid oxygen per hour. Courtesy of Essex Industries. 


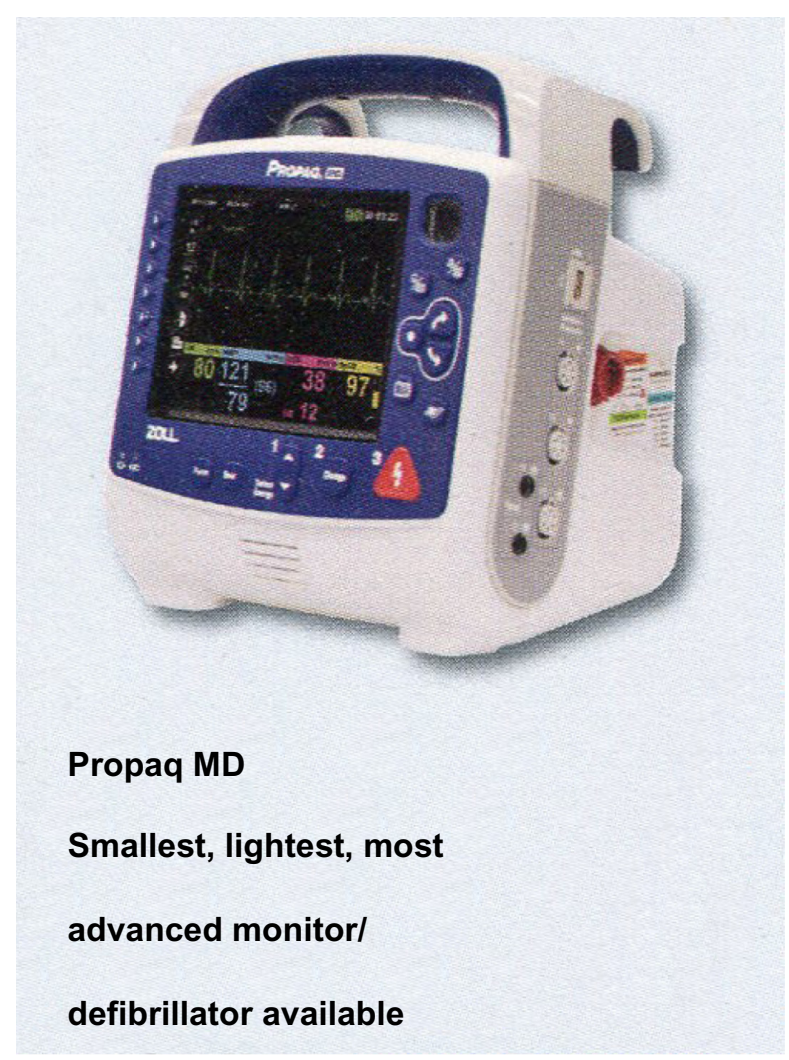

Figure 3 Portable patient monitor and defibrillator. Courtesy of Zoll Medical Corporation.

developed by NeuroLogica Corporation (Danvers, MA, USA, see Figure 4). The CereTom CT scanner has all basic capabilities (contrast CT, CT angiography, and xenon perfusion $\mathrm{CT}$ ), can be easily moved on rollers by one person, and can be powered by a 12 volt car battery using an inverter. A larger but still portable version for body CT scanning (BodyTom $^{\circledR}$, NeuroLogica Corporation) is also available. Figure 4B and C illustrate the BodyTom CT scanner in both operational (Figure 4B) and transport mode in a containerized imaging room/operating room that can be air-lifted to the disaster site (as shown in Figure 1). Now that NeuroLogica Corporation has recently been acquired by Samsung Corporation, there should be availability and support worldwide.

Perhaps the most difficult resource to have immediately at the site of a disaster is the medical specialist, such as a neurosurgeon. One solution for this is telemedicine, allowing the remotely located medical specialist to be "virtually" present at the disaster site. For a surgeon, this remote presence should go beyond merely having visual and auditory contact with those medically managing the disaster scene. Surgical procedures unfamiliar to the emergency response team can often be managed if the medical specialist (eg, trauma neurosurgeon) can act as a "virtual surgical assistant". One device
A
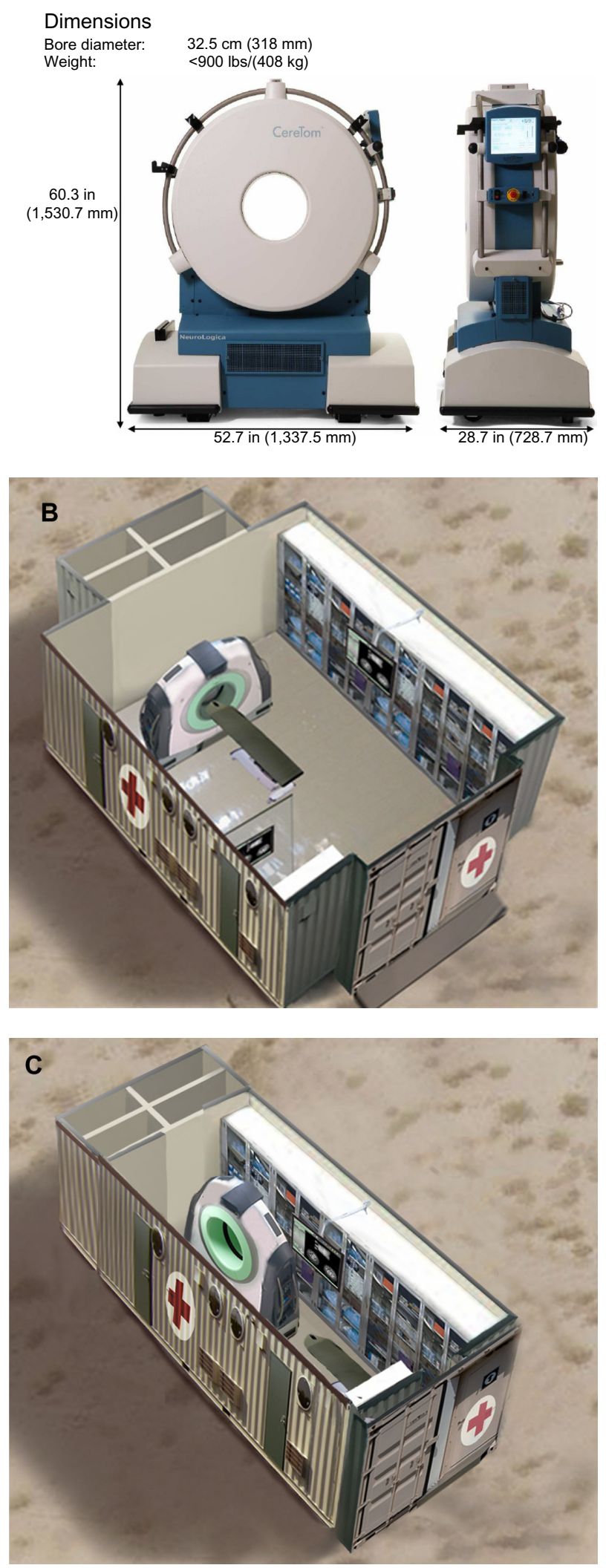

Figure 4 CereTom $^{\circledR}$ portable computed tomography scanner (A). Rendering of BodyTom portable CT scanner in expanded operational mode (B). Rendering of portable CT scanner container in transport mode (C).

Note: Images courtesy of NeuroLogica. 
being developed for this purpose by Vigilent Telesystems (Dorval, QC, Canada) consists of two remotely controlled robotic arms, each with a camera, which can be mounted on the ceiling of a remote clinic or a portable mobile emergency unit operating room (Figure 5A and B). A trauma neurosurgeon, perhaps thousands of miles away, is able to control the cameras and interact verbally with the medical personnel at the disaster site, much as an attending surgeon might supervise a junior colleague in training. A portable, briefcase-sized, battery-powered, remotely controlled camera is being developed to allow continuous visual monitoring of, eg, a trauma patient from the trauma site, in the ambulance or helicopter, to the hospital and operating room. The remotely located medical/surgical specialist could thus monitor and direct the care of a trauma patient from the scene of injury to definitive inhospital treatment at either a medical center or a disaster response mobile emergency unit. Such a portable remote monitoring device is functional wherever there is Internet access.
Perhaps the best example of a major telemedicine program developed with efficient use of resources in mind is the Apollo Telemedicine Networking Foundation (ATNF), which is part of the Apollo Healthcare System in India. The ATNF president, Krishnan Ganapathy, is a neurosurgeon in Chennai who has spearheaded the development of a telemedicine system to support health care not only throughout India, but also to provide teleconsulting services to other countries in the region, and additionally to over 20 sub-Saharan African countries. ${ }^{6}$ The dramatic benefit of such a telemedicine program in minimizing the loss of life in a natural disaster is demonstrated in the following section.

\section{Disaster prevention}

Clearly prevention is superior to treatment in disasters, as in medicine in general. There are effective programs to educate people on risky behaviors, from poor eating habits to smoking to risky sexual activity. Relevant to disasters
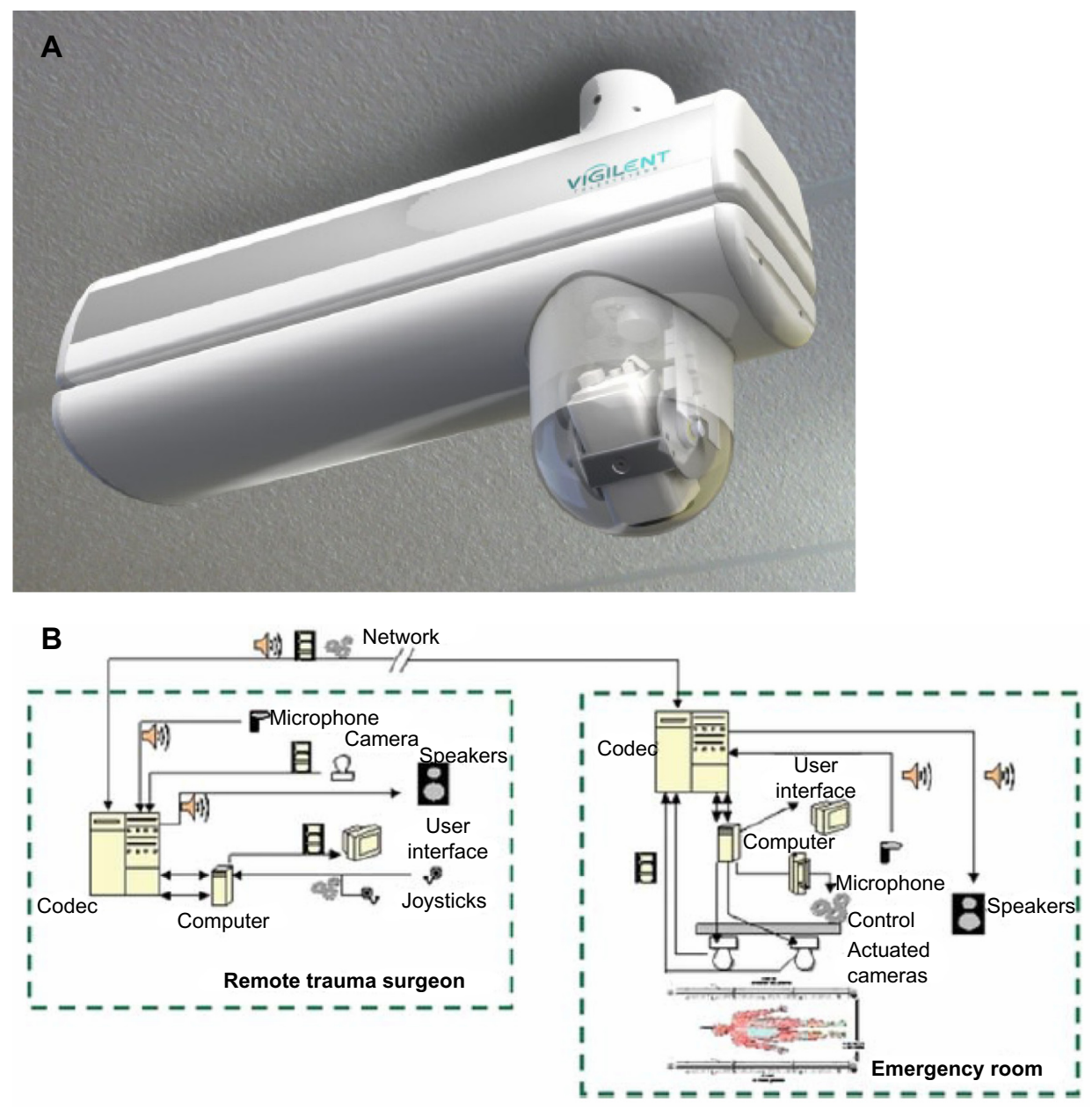

Figure 5 Remote control robotic cameras (Vigilent Telesystems, Dorval, QC, Canada). (A) Ceiling-mounted camera in a remote clinic/emergency room and (B) schematic of the system. Courtesy of Vigilent Telesystems. 
Table I Comparison of recent cyclones/typhoons in South and Southeast Asia

\begin{tabular}{lllll}
\hline Name & Country & Date & $\begin{array}{l}\text { Maximum } \\
\text { wind speed }\end{array}$ & $\begin{array}{l}\text { Estimated } \\
\text { deaths }\end{array}$ \\
\hline Nargis & Myanmar & April 2008 & $215 \mathrm{~km} / \mathrm{hour}$ & $\sim 140,000$ \\
Phailin & India & October 2013 & $260 \mathrm{~km} / \mathrm{hour}$ & $<50$ \\
Haiyan & Philippines & November 2013 & $315 \mathrm{~km} / \mathrm{hour}$ & $\sim 7,000$ \\
\hline
\end{tabular}

Note: data from Kim and Chan, ${ }^{4}$ Wikipedia, ${ }^{5}$ and Bollineni. ${ }^{6}$

are trauma-oriented programs such as the ThinkFirst Injury Prevention Foundation, which has developed publications and programs to educate young people (from kindergarten through high school) on how to minimize the risk of traumatic injury in daily life. ${ }^{?}$

Although it is not feasible to expect prevention of most natural disasters such as earthquakes and cyclones/ hurricanes/typhoons, nor perhaps manmade disasters such as terrorist attacks and aircraft/building/ship/train accidents, it is reasonable to employ all available resources to minimize the loss of life resulting from such disasters. Improved building standards and educating the populace, for example, can greatly reduce the suffering inflicted by an earthquake.

In the case of cyclones/hurricanes/typhoons (where there is more advanced warning than for earthquakes), one should not underestimate the value of coordinated planning and response at the time the disaster is imminent. One might compare three recent severe storms affecting regions of south and southeast Asia, ie, Cyclone Nargis, Cyclone Phailin, and Typhoon Haiyan (Table 1). ${ }^{8-10}$ The difference in loss of life between these three severe storms is quite amazing: an estimated 138,000 in Cyclone Nargis, more than 7,000 in Typhoon Haiyan, but less than 50 in Cyclone Phailin. Although Cyclone Nargis was the weakest in terms of wind speed, the storm surge up the Irrawaddy Delta caused devastating loss of life. In both Cyclone Nargis and Typhoon Haiyan, the vast majority of deaths were caused by storm surges rather than wind or wind damage.

But how can one account for the amazingly low number of deaths from Cyclone Phailin? A good portion of the credit must go to the long-term governmental planning and immediate action preceding Cyclone Phailin's landfall in the state of Odisha on India's east coast. This was facilitated by the network of 31 telemedicine stations established throughout the state of Odisha. Estimates of the number of people evacuated from low-lying areas range up to $1,300,000$, and evacuees were housed in 600 storm shelters. ${ }^{9}$ One wonders what the loss of life in either Cyclone Nargis or Typhoon Haiyan would have been if a similar pre-emptive evacuation had been undertaken, as was the case with Cyclone Phailin.
Just as it is hard to overestimate the benefits of planning for predisaster life-saving interventions (such as the coordinated evacuation for Cyclone Phailin), it is hard to overestimate the benefits of planning for rapid post-disaster deployment of medical personnel.

\section{Integration is key to implementation of the disaster response}

To estimate the benefits of immediate disaster response, one need only look at the benefits of the trauma center system, integrated into the ongoing medical care delivery and training system, that has evolved in the USA and elsewhere. ${ }^{1}$ Compare this with the response to the devastating earthquake of January 2010 in Haiti, where over 100,000 people died. Reportedly the first international response team to arrive in Port-au-Prince, and perhaps the only such team to arrive within 24 hours of the earthquake, was the Icelandic Association for Search and Rescue. ${ }^{11}$ It was estimated by Ophelia Dahl, Executive Director of Partners for Health, that during the first week after the earthquake, as many as 20,000 people who could have been saved by surgery died each day. ${ }^{12}$

Here we concentrate on the potential for neurosurgical organizations worldwide to provide an outline of how an immediate disaster response might be integrated into the medical care delivery and training system, much like the trauma center system has been integrated into the medical system in the USA. Clearly, many other national and international organizations and governments would need to participate to improve disaster response on a global scale, and would include:

- trauma organizations, eg, American Trauma Society

- traditional medical/surgical societies

o national societies, eg, American Association of Neurological Surgeons

o international societies, eg, World Federation of Neurosurgical Societies

- related societies

o medical resources, eg, Aerospace Medical Association

o medical personnel, eg, Médecins Sans Frontières

- nongovernmental organizations

o injury prevention, eg, ThinkFirst Foundation

o physician participation, eg, Foundation for International Education in Neurological Surgery

- governmental/military: eg, US Army Medical Department

- international organizations, eg, Red Cross, UNOCHA.

The primary global neurosurgical organization is the World Federation of Neurosurgical Societies (WFNS), which 
consists of five continental neurosurgical associations, eg, the European Association of Neurosurgical Societies (EANS), 114 national neurosurgical societies, and five affiliate societies, in total involving 30,000 neurosurgeons worldwide. To quote from the WFNS website: ${ }^{13}$

The WFNS aspires to promote global improvement in neurosurgical care. The mission of the WFNS is to work together with our member societies to improve worldwide neurosurgical care, training and research to benefit our patients.

To date, neurosurgical training and certification for practice as a neurosurgeon has taken place at the country level. An exception to this has been the creation by the WFNS of regional international training centers such as the WFNS-sponsored neurosurgical training center for young African neurosurgeons in Rabat, Morocco, that began in 2002 .

The EANS has taken the lead in unifying neurosurgical education, training, and certification by offering courses that provide continuing medical education throughout the European Union as well as written and oral certification examinations (similar to the US board certification process) that are also recognized throughout the European Union. Individual neurosurgical training programs anywhere in the European Union can apply to become an EANS-accredited neurosurgical training program.

\section{Existing barriers to integration in the USA}

Contrast the situation in the European Union described above with the situation in the USA, where the Accreditation Council for Graduate Medical Education (ACGME) establishes the criteria for post-medical school specialty training programs (like neurosurgery), and the American Board of Neurological Surgery (ABNS) establishes the criteria for granting "board certification" to individual neurosurgeons. The only training programs in neurosurgery outside the USA recognized by the ABNS as worthy of permitting their graduates to apply for ABNS board certification were those training programs in Canada, and beginning in 1997, even neurosurgeons graduating from Canadian neurosurgical training programs were no longer recognized for board certification by the $\mathrm{ABNS}$. To meet $\mathrm{ABNS}$ requirements, a neurosurgery training program must be ACGME-accredited (thus limiting the programs to those in the USA) and the 54 "core" months of the total 84-month minimum training program (ie, 30 months are "elective") must take place in an ACGME-accredited program. To quote from the ABNS website: ${ }^{14}$

The ABNS may [...] grant elective credit for training in non-ACGME accredited US or foreign centers on an individual basis [...]. Approval for training periods of less than three months will not ordinarily be granted since they are of questionable educational value.

For a neurosurgeon in training in the USA to participate in a training program outside that country (only during the 30 elective months, not the 54 core months), it is necessary to first secure approval from the ABNS and then approval from the ACGME. The ACGME international rotation application process for neurosurgery includes 13 points, only one of which is listed here:

"Rotation description, including:

- competency-based goals and objectives

- verification that the rotation is elective

- verification that the experience will include an outpatient experience

- how the rotation will ensure that the resident participates in continuity of care (pre-, peri-, and post-operative care)

- how the proposed rotation will provide experience not available at the sponsoring institution or current participating sites

- how patients will be followed by local staff members after the resident has completed the rotation, and how the resident will receive feedback about individual patient outcomes

- a plan for resident assessment during the rotation (must include at least one mid-rotation and an end-of-rotation assessment)." 15

It is certainly essential to ensure quality in all training experiences, but the requirements for a program outside the USA to be permitted to accept an in-training neurosurgeon from the USA for a portion of his/her overall neurosurgery training are extremely burdensome. This is likely a major reason why so few US in-training neurosurgeons gain experience in training programs outside the USA.

There are barriers in the other direction also, ie, for a neurosurgeon in training from outside the USA to come to a neurosurgery training program in the USA for a clinical experience. Somewhat ironically, a medical student from any country can participate in "hands-on" clinical activities, notably interacting with patients in the outpatient clinic and in hospital, as well as "scrubbing in" on surgical procedures. Medical students are deemed to be under the direct supervision of the attending or consultant neurosurgeon. Once an in-training neurosurgeon 
has reached the stage of medical licensure in the USA (ie, following medical school), he/she, despite being under similar direct supervision by the attending or consultant neurosurgeon, cannot participate in "hands-on" clinical activities. Thus in the USA, these non-US neurosurgeons in training are limited to laboratory research activities or observerships, unlike the situation in the European Union and elsewhere.

Even decades ago, but certainly today, there are many neurosurgery training programs around the world that are on a par with the ACGME-accredited programs in the USA. It is time to take a model such as the EANS has done for neurosurgery training and certification in the European Union and expand it worldwide.

\section{Integrated program for immediate disaster response}

This section describes how improving disaster response can facilitate the WFNS mission "to improve worldwide neurosurgical care, training and research to benefit our patients". Since disaster response presents a rare opportunity to transcend the socioeconomic, legal, and political barriers that impede not only health care delivery but also social harmony worldwide, the "return on investment" of this project could be very great indeed. As demonstrated in the section above, to have a significant impact on reducing the loss of life in disasters, a disaster response program must include the following:

- mobilization to the disaster site within 24 hours

- transport of all necessary resources (mobile emergency unit, generators, supplies, personnel), requiring a large helicopter (eg, Chinook, which has a payload of 12,000 kg)

- resources ideally located within $\sim 1,000 \mathrm{~km}$ of the disaster site (Chinook helicopter range $\sim 1,100 \mathrm{~km}$; single refueling stop $\sim 2,200 \mathrm{~km}$ range).

To meet these logistics requirements, it would be necessary to have at least four disaster response centers (DRCs) worldwide, ready at a moment's notice, to proceed by helicopter transport to the disaster site. Although disaster response is by no means adequate in developed countries, the magnitude of the problem is much greater in other regions of the world. Thus, DRCs would ideally be located in Latin America, Africa, Central Asia, and Southeast Asia. Admittedly only four DRCs would not allow a DRC to be placed within $\sim 2,000 \mathrm{~km}$ of every potential disaster site (particularly in the case of Africa), but four such strategically located DRCs would vastly improve the response to most disasters worldwide, and serve as an impetus for additional
DRCs to be formed once the benefits of such DRCs are appreciated.

The DRC project will obviously need administrative, political, and financial support from such international organizations as the WFNS, World Health Organization, national medical/surgical societies, governments (such as the support for the trauma center system in the USA), and nongovernmental organizations such as Médecins Sans Frontières, the ThinkFirst Foundation, and private foundations supporting health care in developing countries (eg, the Bill and Melinda Gates Foundation and the Clinton Foundation). But equally important for reasonably rapid implementation as well as long-term viability is the presence of a basic local health care infrastructure and personnel dedicated to the success of the DRC.

A DRC site would need to have an ongoing medical center with a reasonably well developed infrastructure and training programs in place. It will also need to have individuals with a commitment to seeing through the planning, establishment, and maturation phases of a DRC site. Two locations that meet these criteria are Iquique in northern Chile and Peshawar in northern Pakistan. In the case of Iquique, one of the authors (LQ) has familiarity with the local resources (eg, neurosurgery and trauma) in Iquique as well as having established contact with the Chilean Ministry of Health to support the telemedicine and other infrastructure items necessary to expand the capabilities of the medical center in Iquique to become a DRC. In the case of Peshawar, Tariq Khan, a neurosurgeon who is a native of that region, constructed a full-service hospital in Peshawar, and is one of the senior members of the WFNS neurotraumatology committee, and is well suited to oversee the creation of a DRC. Peshawar, although not strictly part of Central Asia, is reasonably located to provide timely disaster response to most of Central Asia as well as the remote regions of western China and much of western South Asia.

From the neurosurgical aspect, creation of a DRC site would begin with application to the WFNS for a DRC designation. The proposed DRC site would need to have ongoing clinical services that could be developed into a DRC site, including neurosurgery, trauma/critical care, and orthopedics. In order to support the WFNS goals of improving international neurosurgical education and the standardization of neurosurgical training worldwide, the proposed DRC site would need to have a training program that meets international standards. The EANS model for approving neurosurgical training sites throughout the 
European Union could be used as a starting point for accreditation.

One key component would be staffing of the DRC that includes physicians (both faculty and in training) and other health care professionals not only from the DRC host site and country, but also from other countries, including both neighboring countries and more distant, more developed countries such as in Europe, parts of Asia, and the USA and Canada. Neurosurgery faculty from developed countries might spend a period at the DRC from one month to one year, or longer. Such temporary placement of neurosurgeons from developed countries into developing countries has been fostered for years by organizations such as the Foundation for International Education in Neurosurgery. Elaborating on the model of the WFNS-sponsored program in Rabat for training neurosurgeons from underserved and underdeveloped African countries, there could be exchanges of in-training neurosurgeons between the DRC and training programs in developed countries. These exchanges might also last from one month to one year, although following the ABNS recommendation, a period of at least 3 months would be desirable. A post-trauma rehabilitation team, again including both local and international physicians and support staff, together with clinical and research rehabilitation facilities, would be another integral part of the DRC. Similar to a trauma center, there must be a team on call at all times for immediate deployment to a disaster site.

Crucial to the success of a DRC are the physical as well as personnel resources necessary to create and sustain the DRC. Several large helicopters would need to be available for use on short notice, ie, within a few hours. The fully equipped mobile emergency unit operating room, as documented earlier, would also need to ready for immediate transport. It is possible (and indeed desirable from a personnel familiarity standpoint) that an mobile emergency unit would be in occasional if not constant use by the DRC team. Not only could the mobile emergency unit provide additional surgical/ operating room resources for the DRC, it could also serve as a surgical training and research facility.

\section{Benefits of the disaster response center program}

The obvious benefit of a much more immediate medical response to disasters is the reduction in mortality and morbidity. However, when the DRC is integrated into the larger disaster planning program of a region or country, the benefits become much more pervasive. As was seen with Cyclone Phailin, the combination of an effective telemedicine network and long-range planning for largescale evacuation of people at risk can greatly reduce the loss of life in disasters where at least some warning is available.

Equally important, however, are the benefits of the integrated DRC program beyond those directly related to the human toll of disasters. A DRC in a medically underserved region will enhance the daily medical care of the people in that region, particularly when the benefits of a telemedicine program for those in remote regions is considered. Again, the ATNF in India is pioneering the use of cost-effective telemedicine to bring health care to remote areas, eg, the use of smartphones to transmit point-of-care diagnostics, such as electrocardiograms and blood tests.

Socioeconomic benefits will accrue as well. Not only will the local government be likely to support the DRC (given its benefit for the health of the citizens in the region noted above), but international public health agencies and private foundations concerned with global health care will see the advantages of the DRC as both a clinical and research platform for augmenting cost-effective health care worldwide. Given the infrastructure development and academic underpinnings of the DRC, medical schools and schools of public health in developed countries will also see an opportunity for costeffective clinical and basic research.

There are long-term benefits for the companies that support health care, from pharmaceutical companies to medical device and equipment manufacturers. Since many markets for medical equipment and drugs are approaching saturation in developed countries, the ability of a DRC to "fast forward" the development of health care in the region will not be overlooked by these companies. Because of the public relations benefits that accrue from supporting such a humanitarian effort as the DRC, these medical companies will likely be eager to donate equipment and supplies to the DRC, at least in the initial stages. In many cases, the favorable tax consequences of such humanitarian donations of medical equipment and supplies are very persuasive as well.

Finally, there are the educational and social benefits of the DRC. For neurosurgery and other medical specialties, the DRC can be a relatively "apolitical" means by which to foster universal standards for the training and certification of new physicians. The exchange of junior (in-training) neurosurgeons, for example, between a developing country (where the DRC is located) and a developed country will establish both a mutual respect and a camaraderie that can 
be invaluable in finding mutually acceptable solutions when differences arise between individuals (and countries) with different backgrounds. Although such exchanges are usually felt to benefit the junior physician coming to the developed country medical center (to learn about the latest high-tech medical devices and procedures), perhaps an even greater benefit accrues to the junior physician leaving those high-tech medical devices and procedures behind. Lacking (in the case of a neurosurgeon) intraoperative magnetic resonance imaging and gigabytes of digital diagnostic information (both imaging and laboratory studies), the physician is forced to rely on those nearly forgotten aspects of medicine, ie, the history and physical examination.

It was demonstrated above how the ATNF in India has taken a relatively basic telemedicine system and made it useful for health care throughout India and as far afield as sub-Saharan Africa. This theme of innovative use of technology that is available worldwide to benefit the health of hundreds of millions of people in developing countries, ie, learning from those in the developing world on how best to tackle major health care problems, rather than assuming the developed country answer is the only choice, has been described recently. Nigel Crisp, who ran the National Health Service in the UK from 2000 to 2006, has since cataloged the many ways health care in developed countries might learn from developing countries. ${ }^{16}$ The DRC program will no doubt learn a great deal from health care professionals in developing countries, and the program will likely look much different as it matures.

Some of the benefits of the DRC program are as follows:

- reduction in disaster morbidity and mortality due to much more rapid treatment

- reduction in disaster morbidity and mortality due to improved disaster planning, eg, telemedicine and evacuation

- improved ongoing health care for people living in the region of the DRC (improvement in both medical/surgical resources and personnel as well as telemedicine for more remote areas)

- support from local/national government, international public health agencies, and private foundations concerned with global health care

- involvement by medical/pharmacy/nursing/rehabilitation training centers/universities from developed countries

- equipment/drugs/supplies donated by companies that realize the long-term benefits of helping to develop health care in underserved regions
- worldwide standardization of training and certification criteria for physicians, nurses, and other health care personnel

- enhanced mutual understanding of the advantages and limitations of health care systems in developing and developed countries by physicians and other health care professionals in training, thanks to training program exchanges

- the camaraderie that develops from in-training individuals of different cultural backgrounds working side by side on a daily basis.

In this era when communication (cell phones), money (banking), and people (air travel) are moved seamlessly around the world, it is an anachronism to have health care delivery and medical training remain in its present largely balkanized state. The DRC program, because of the humanizing (although devastating) effect of disasters, can be a means to bring health care into the "global village" of the 21 st century.

\section{Disclosure}

The authors report no conflicts of interest in this work. The views expressed are those of the authors, and not the official policy of the US government, the National Aeronautics and Space Administration, or the World Federation of Neurosurgical Societies.

\section{References}

1. Haas B, Stukel TA, Gomez D, et al. The mortality benefit of direct trauma center transport in a regional trauma system: a population-based study. J Trauma Acute Care Surg. 2012;72(6):1510-1517.

2. United Nations Office for the Coordination of Humanitarian Affairs. Available from: http://www.unocha.org/. Accessed January 14, 2014.

3. GlobalCorps. List of Relief Organizations. Available from: http://www. globalcorps.com/jobs/ngolist.pdf. Accessed January 17, 2014.

4. Kim JY, Chan M. Poverty, health, and societies of the future. JAMA. 2013;230(9):901-902.

5. Wikipedia. 2010 Copiapó mining accident. Available from: http:// en.wikipedia.org/wiki/2010_Copiap\%C3\%B3_mining_accident. Accessed February 4, 2014.

6. Bollineni R. Apollo Telemedicine Networking Foundation. Center for Health Market Innovations and Access Health International, 2011. Available from: http://healthmarketinnovations.org/sites/default/files/ Apollo\%20Case\%20Study.pdf. Accessed February 4, 2014.

7. ThinkFirst National Injury Prevention Foundation. Available from: http://www.thinkfirst.org. Accessed December 6, 2013.

8. International Federation of Red Cross and Red Crescent Societies. Myanmar: Cyclone Nargis 2008 Facts and Figures. Available from: http://www.ifrc.org. Accessed March 30, 2014.

9. Government of India, India Meteorological Department. Cyclonic Storm PHAILIN over the Bay of Bengal: A Report. Available from: http:// www.imd.gov.in/section/nhac/dynamic/phailin.pdf. Accessed March 30, 2014.

10. Republic of the Philippines, National Disaster Risk Reduction and Management Council. Effects of Typhoon Yolanda (Haiyan) [NDRRMC Update SitRep No. 107, 14 March 2014]. Available from: http://www. ndrrmc.gov.ph. Accessed March 30, 2014. 
11. Nordic World AS. The Icelandic Search and Rescue Teams: Haiti Response. Available from: http://www.nordicworld.tv. Accessed March $30,2014$.

12. CBS News 60 Minutes 18 Jan 2010. @katiecouric: Disaster in Haiti. Available from: http://www.cbsnes.com/videos/katiecouric-disaster-inhaiti/. Accessed March 30, 2014.

13. World Federation of Neurosurgical Societies. Available from: http:// www.wfns.org. Accessed December 6, 2013.

14. American Board of Neurological Surgery. Neurosurgery residency training. Available from: http://www.abns.org/content/primary_ certification_process.asp. Accessed January 4, 2014.
15. Accreditation Council for Graduate Medical Education Review Committee for Neurological Surgery. International rotation application process. Available from: http://www.acgme.org/acgmeweb/Portals/0/ PFAssets/ProgramResources/160_International_Rotation_Application_ Process.pdf. Accessed 4 January 2014.

16. Crisp N. Turning the World Upside Down: The Search for Global Health in the 21st Century. Boca Raton, FL, USA: CRC Press; 2010.

Journal of Neurorestoratology

\section{Publish your work in this journal}

The Journal of Neurorestoratology is an international, peer-reviewed, open access online journal publishing original research and review articles on the subject of Neurorestoratology. To provide complete coverage of this revolutionary field the Journal of Neurorestoratology will report on relevant experimental research, technological advances, and

clinical achievements. The manuscript management system is completely online and includes a very quick and fair peer-review system, which is all easy to use. Visit http://www.dovepress.com/testimonials.php to read real quotes from published authors. 